\title{
Extremal Vector Valued Inequalities for Hankel Transforms
}

\author{
Elena ROMERA
}

\author{
Departamento de Matemáticas \\ Universidad Carlos III de Madrid \\ Avenida de la Universidad, 30 \\ 28911 Leganés (Madrid) — Spain \\ eromera@math.uc3m.es
}

Received: February 15, 2008

Accepted: June 5, 2008

\section{ABSTRACT}

The disc multiplier may be seen as a vector valued operator when we consider its projections in terms of the spherical harmonics. In this form, it represents a vector valued Hankel transform. We know that, for radial functions, it is bounded on the spaces $L_{l q}^{p}\left(r^{n-1} d r\right)$ when $\frac{2 n}{n+1}<p, q<\frac{2 n}{n-1}$. Here we prove that there exist weak-type estimates for this operator for the extremal exponents, that is, it is bounded from $L_{l q}^{p_{i}, 1}\left(r^{n-1} d r\right)$ to $L_{l q}^{p_{i}, \infty}\left(r^{n-1} d r\right)$ for $i=0,1$ when $p_{0}=\frac{2 n}{n+1}, p_{1}=\frac{2 n}{n-1}, p_{0}<q<p_{1}$, and we consider radial functions.

Key words: disc multiplier, Fourier-Hankel transforms.

2000 Mathematics Subject Classification: 42B10.

\section{Introduction and statement of theorems}

Let us call $T$ the disc multiplier operator, given by

$$
\widehat{(T f})(\xi)=\chi_{B(0,1)}(\xi) \hat{f}(\xi),
$$

where $B(0,1)=\{x:|x|<1\}$ and $\chi_{A}$ is the characteristic function of the set $A$. This operator is related to the Hilbert transform. In particular, in one dimension, the multiplier operator of the disc of radius $R$, which we denote by $S_{R}$, is given by

$$
S_{R} f(x)=c\left(e^{i R x} H\left(f(\cdot) e^{-i R \cdot}\right)(x)-e^{-i R x} H\left(f(\cdot) e^{i R \cdot}\right)(x)\right) .
$$

Research partially supported by Comunidad de Madrid-Universidad Carlos III (CCG07-UC3M/ESP$3339)$. 
Here $H$ is the Hilbert transform, defined by

$$
H f(x)=\text { p.v. } \frac{1}{\pi} \int_{\mathbb{R}} \frac{f(y)}{x-y} d y .
$$

With this notation, $T=S_{1}$, and the boundedness of $T$ may be obtained from the boundedness of $H$ because the difference is only the conjugation with functions of norm one. But we know much more about $T$, because in 1966 L. Carleson proved $($ see $[2])$ :

Theorem 1.1 (Carleson). The maximal operator defined by

$$
\sup _{R \in \mathbb{R}}\left|H\left(f(\cdot) e^{i R \cdot}\right)\right|=\sup _{R \in \mathbb{R}} \mid \text { p.v. } \frac{1}{\pi} \int_{\mathbb{R}} \frac{f(y) e^{i R y}}{x-y} d y \mid
$$

is bounded from the space $L^{2}(\mathbb{R})$ to $L^{2}(\mathbb{R})$.

Later on, in 1968, R. Hunt (see [6]) extended this theorem to the spaces $L^{p}(\mathbb{R})$ with $1<p<\infty$.

When we consider now dimension $n$, the analogous decomposition of $T$ is obtained from the expansion in spherical harmonics of a function of $L^{2}\left(\mathbb{R}^{n}\right)$. The expression we obtain for $T$ is very useful because of the good behavior of these polynomials with respect to the Fourier transform. This decomposition is obtained as follows.

Let $\left\{\mathcal{Y}_{j}^{(k)}\right\}_{k \geq 0,1 \leq j \leq a_{k}}$ be an orthonormal basis of spherical harmonics in $L^{2}\left(S^{n-1}\right)$. Each one of the functions $\mathcal{Y}_{j}^{(k)}$ is the restriction to the sphere of a homogeneous harmonic polynomial of degree $k$ and $a_{k}$ is the dimension of the space $A_{k}$ of all the homogeneous harmonic polynomials of degree $k$ (see the reference [12]).

A function $f$ in $L^{2}$ has the following expansion in terms of that basis:

$$
f(x)=\sum_{\substack{k \geq 0 \\ 1 \leq j \leq a_{k}}} f_{k, j}(|x|) \mathcal{Y}_{j}^{(k)}\left(\frac{x}{|x|}\right),
$$

where the functions $f_{k, j}$ are the coefficients. The Fourier transform of such a function is then

$$
\begin{aligned}
\hat{f}(\xi) & =\sum_{k, j} \frac{i^{-k}}{|\xi|^{\frac{n-2}{2}}} \int_{0}^{\infty} f_{k, j}(t) J_{\frac{n-2}{2}+k}(|\xi| t) t^{\frac{n}{2}} d t \mathcal{Y}_{j}^{(k)}\left(\frac{\xi}{|\xi|}\right) \\
& =\sum_{k, j} \frac{i^{-k}}{|\xi|^{\frac{n-2}{2}}} H_{\frac{n-2}{2}+k}\left(f_{k, j}(s) s^{\frac{n-2}{2}}\right)(|\xi|) \mathcal{Y}_{j}^{(k)}\left(\frac{\xi}{|\xi|}\right)
\end{aligned}
$$

where $J_{l}$ is the Bessel function of order $l$ and $H_{l}$ is the Fourier-Hankel transform, also of order $l$, which we define for functions $g:(0, \infty) \rightarrow \mathbb{C}$ by the formula

$$
H_{l} g(r)=\int_{0}^{\infty} g(s) J_{l}(r s) s d s .
$$


[The definition of Fourier transform we are using here is

$$
\hat{f}(\xi)=\frac{1}{(2 \pi)^{n / 2}} \int_{\mathbb{R}^{n}} e^{-i x \xi} f(x) d x
$$

which makes the notation simpler.]

The corresponding formula for the disc multiplier in terms of the spherical harmonics is

$$
T f(x)=\sum_{k, j} \frac{1}{|x|^{\frac{n-1}{2}}} T_{\frac{n-2}{2}+k}\left(f_{k, j}(s) s^{\frac{n-1}{2}}\right)(|x|) \mathcal{Y}_{j}^{(k)}\left(\frac{x}{|x|}\right),
$$

where $T_{l}$ denotes the operator

$$
T_{l} g(r)=\int_{0}^{\infty} g(s)(r s)^{1 / 2} \int_{0}^{1} J_{l}(t r) J_{l}(t s) t d t d s .
$$

With this formula in mind we can see $T$ as an operator acting on sequences of functions. Those functions are the coefficients of $f$ in terms of the spherical harmonics.

In general, given a sequence of positive numbers, $a=\left\{l_{k}\right\}_{k}$, each $l_{k}$ greater than a fixed positive constant, we can define the vector valued operator $\widetilde{T}\left(=\widetilde{T}_{a, n}\right)$, acting on a suitable sequence of functions $\left\{f_{k}\right\}_{k}$, defined in $(0, \infty)$, by the formula

$$
\widetilde{T}\left(\left\{f_{k}\right\}_{k}\right)(r)=\left\{\frac{1}{r^{\frac{n-1}{2}}} T_{l_{k}}\left(f_{k}(s) s^{\frac{n-1}{2}}\right)(r)\right\}_{k}, \quad r>0 .
$$

For this operator we already know that it is bounded when acting on some of the spaces $L_{l q}^{p}\left(r^{n-1} d r\right)$ that we define as the spaces of sequences of radial functions $\left\{f_{k}\right\}_{k}$ in $l^{q}$ such that

$$
\left\|\left\{f_{k}\right\}_{k}\right\|_{L_{l q}^{p}\left(r^{n-1} d r\right)}=:\left(\int\left(\sum_{k}\left|f_{k}\right|^{q}\right)^{p / q} r^{n-1} d r\right)^{1 / p}=\left\|\left|\left\{f_{k}\right\}_{k}\right|_{l^{q}}\right\|_{L^{p}\left(r^{n-1} d r\right)}<\infty .
$$

First of all, we know that $\widetilde{T}$ is bounded on the spaces $L_{l^{2}}^{p}\left(r^{n-1} d r\right)$ for $\frac{2 n}{n+1}<p<\frac{2 n}{n-1}$. This estimate is equivalent to the boundedness of the disc multiplier in the mixed norm spaces $L_{\text {rad }}^{p} L_{\text {ang }}^{2}\left(\mathbb{R}^{n}\right)$ for the same rank of $p$, where $L_{\text {rad }}^{p} L_{\text {ang }}^{2}\left(\mathbb{R}^{n}\right)$ is the space of functions defined on $\mathbb{R}^{n}$ with radial part in $L^{p}$ and angular part in $L^{2}$.

This estimate for the disc multiplier was proved independently by A. Córdoba [3] (in a paper of the year 1989) and G. Mockenhaupt [7] (in a paper of the year 1990). A little bit later (in a paper of the year 1992) this result was also obtained as a corollary of the main theorem on weighted inequalities in $L^{2}$ for the disc multiplier by A. Carbery, F. Soria, and the author [1], a problem suggested by E. Stein [11] and solved there for the case of radial weights. Moreover, in [1] we also obtain extremal bounds for the operator $\tilde{T}$ between the spaces

$$
\widetilde{T}: L_{l^{2}}^{p_{i}, 1}\left(r^{n-1} d r\right) \longrightarrow L_{l^{2}}^{p_{i}, \infty}\left(r^{n-1} d r\right), \quad i=0,1,
$$


where $p_{0}=\frac{2 n}{n+1}, p_{1}=\frac{2 n}{n-1}$, and $n \geq 2$. These estimates also have an interpretation as mixed norm estimates for the disc multiplier $T$, in particular, it is bounded between the spaces

$$
T: L_{\mathrm{rad}}^{p_{i}, 1} L_{\text {ang }}^{2}\left(\mathbb{R}^{n}\right) \longrightarrow L_{\mathrm{rad}}^{p_{i}, \infty} L_{\mathrm{ang}}^{2}\left(\mathbb{R}^{n}\right), \quad i=0,1 .
$$

These are weak type estimates but, nevertheless, they imply the previous bounds using interpolation and so, they extend the previous result.

With different techniques from those used in the case of $L_{l^{2}}^{p}$, which involve weighted estimates and interpolation, it is proved in [9] that the operator $\widetilde{T}$ is bounded on the spaces $L_{l^{q}}^{p}$ with $p_{0}<p, q<p_{1}$ when acting on radial functions.

Let us consider also the operator $Q$ obtained by eliminating in the expression of $\widetilde{T}$ the conjugation with $s^{\frac{n-1}{2}}$ :

$$
Q\left(\left\{f_{k}\right\}_{k}\right)(r)=\left\{T_{l_{k}} f_{k}(r)\right\}_{k}, \quad r>0
$$

For this new operator we know that it is bounded in the space $L_{l^{2}}^{p}(d r)$ for $\frac{4}{3}<p<4$. This fact is the basic step of A. Córdoba in the proof of the mixed norm estimate for the operator $T$ (see [3]). This estimate was also obtained in [1] by different methods which allowed the authors to obtain the following extremal estimate for $Q$ :

$$
Q: L_{l^{2}}^{4 / 3,1}(d r) \longrightarrow L_{l^{2}}^{4 / 3, \infty}(d r)
$$

In the reference [9] the author proves also that the operator $Q$ is bounded from $L_{l^{q}}^{p}(d r)$ to $L_{l^{q}}^{p}(d r)$ for every $\frac{4}{3}<p, q<4$.

The estimates in the spaces $L_{l q}^{p}$ with $q \neq 2$ are related to some extent with the vector valued inequalities for the Hardy-Littlewood maximal operator of C. Fefferman and E. Stein (see [4]).

In this paper we extend the estimates for $\widetilde{T}$ in $L_{l^{q}}^{p}$ to the extremal exponents obtaining weak type bounds. Our theorem is the following:

Theorem 1.2. For $n>2$, the operator $\widetilde{T}$ is bounded between the spaces

$$
\widetilde{T}: L_{l^{q}}^{p_{i}, 1}\left(r^{n-1} d r\right) \longrightarrow L_{l^{q}}^{p_{i}, \infty}\left(r^{n-1} d r\right), \quad i=0,1
$$

for radial functions, where $p_{0}=\frac{2 n}{n+1}, p_{1}=\frac{2 n}{n-1}$, and $p_{0}<q<p_{1}$.

This result extends the main theorem proved in [9], that now can be obtained using interpolation.

Hint on notation: along the paper, the letter $c$ denotes a constant, which may be different from line to line. 


\section{Proof of the main theorem}

First of all, let us recall a few facts about Bessel functions. An estimate of von Lommel (see [13, p. 134]) allows us to write the following expression for the kernel of the operator $T_{k}$ :

$$
\begin{aligned}
2(r s)^{1 / 2} \int_{0}^{1} J_{k}(t r) J_{k}(t s) t d t= & -\frac{\mu_{k}(s) \sigma_{k}(r)}{r-s}+\frac{\mu_{k}(r) \sigma_{k}(s)}{r-s} \\
& -\frac{\mu_{k}(s) \sigma_{k}(r)}{r+s}-\frac{\mu_{k}(r) \sigma_{k}(s)}{r+s} \\
= & \sum_{m=1}^{4} K_{k}^{m}(r, s),
\end{aligned}
$$

in terms of the functions

$$
\begin{array}{ll}
\mu_{k}(t)=t^{1 / 2} J_{k}(t), \quad t>0, \\
\sigma_{k}(t)=t^{1 / 2} J_{k}^{\prime}(t), \quad t>0 .
\end{array}
$$

These functions $\mu_{k}$ and $\sigma_{k}$ are bounded but not independently of $k$. Let's define also

$$
\tau_{k}(t)=\min \left(k^{1 / 6},\left|\frac{t+k}{t-k}\right|^{1 / 4}\right)
$$

for $t>0$. Then, following G. Mockenhaupt [7] (see also [8]), we know there is a constant $c$ independent of $k$ such that

$$
\begin{aligned}
& \left|\mu_{k}(t)\right| \leq c \tau_{k}(t), \quad t>0, \\
& \left|\sigma_{k}(t)\right| \leq c \tau_{k}^{-1}(t)=c \max \left(k^{-1 / 6},\left|\frac{t-k}{t+k}\right|^{1 / 4}\right), \quad t>0,
\end{aligned}
$$

and, moreover, we have the estimate

$$
\left|\tau_{k}(t)\right| \leq c\left(k^{-1 / 6}+\left|\frac{t-k}{t+k}\right|^{1 / 4}\right)^{-1}, \quad t>0 .
$$

Let us define for $m=1,2,3,4$, the operators

$$
P_{k}^{m} g(r)=\frac{1}{r^{(n-1) / 2}} \int_{0}^{\infty} K_{k}^{m}(r, s) g(s) s^{(n-1) / 2} d s, \quad r>0 .
$$

The inequalities we want to prove are the following:

$$
\left\|\left(\sum_{k=1}^{\infty}\left|P_{k}^{m} g_{k}\right|^{q}\right)^{1 / q}\right\|_{L^{p, \infty}\left(r^{n-1} d r\right)} \leq c\left\|\left(\sum_{k=1}^{\infty}\left|g_{k}\right|^{q}\right)^{1 / q}\right\|_{L^{p, 1}\left(r^{n-1} d r\right)},
$$


for $p=p_{0}, p_{1}$, and $m \in\{1,2,3,4\}$.

Let us observe that if we define the operator $P^{m}$ by $P^{m}\left(\left\{g_{k}\right\}_{k}\right)=\left\{P_{k}^{m} g_{k}\right\}_{k}$, $m \in\{1,2,3,4\}$, then, for $m=1,3, P^{m}$ is the adjoint of $P^{m+1}$ with respect to the pairing

$$
\left\langle\left\{g_{k}\right\}_{k},\left\{f_{k}\right\}_{k}\right\rangle=\sum_{k=1}^{\infty} \int_{0}^{\infty} g_{k}(r) \overline{f_{k}(r)} r^{n-1} d r .
$$

Therefore, the inequalities corresponding to $m=1,3$ in the equation (3) for $p=p_{0}$ (and respectively for $p=p_{1}$ ) are formally equivalent to the corresponding inequalities for $m=2,4$ for $p=p_{1}$ (respectively, for $p=p_{0}$ ). We will only study the proof corresponding to $m=1$ for $p=p_{0}, p_{1}$ and will indicate what to do for the case $m=3$.

Given $j \in \mathbb{Z}$, we consider the intervals $I_{j}=\left[2^{j}, 2^{j+1}\right)$ and $I_{j}^{*}=\left[2^{j-1}, 2^{j+2}\right)$. Then, for a fixed $k \geq 1$ we can write

$$
\begin{aligned}
P_{k}^{1} g_{k}(r) & =\sum_{j=-\infty}^{\infty} P_{k}^{1}\left(g_{k} \chi_{I_{j}^{*}}\right)(r) \chi_{I_{j}}(r)+\sum_{j=-\infty}^{\infty} P_{k}^{1}\left(g_{k} \chi_{\left(I_{j}^{*}\right)^{c}}\right)(r) \chi_{I_{j}}(r) \\
& =A_{k}+B_{k},
\end{aligned}
$$

and so $P^{1}\left(\left\{f_{k}\right\}_{k}\right)=\left\{A_{k}\right\}_{k}+\left\{B_{k}\right\}_{k}$.

\subsection{Estimate of $\left\{A_{k}\right\}_{k}$ for $n>2$}

For the weight $w=r^{n-1}$ and the indices $p_{i}=p_{0}, p_{1}$ we can write (from now on, we omit the symbols \{\}$_{k}$ when calculating the norm of a sequence to simplify our expressions)

$$
\begin{aligned}
w\left\{r:\left\|A_{k}\right\|_{l^{q}}(r)>\lambda\right\} & \leq \frac{c}{\lambda^{p_{i}}} \int_{0}^{\infty}\left\|A_{k}\right\|_{l^{q}}^{p_{i}} r^{n-1} d r \\
& =\frac{c}{\lambda^{p_{i}}} \int_{0}^{\infty}\left\|\sum_{j=-\infty}^{\infty} P_{k}^{1}\left(g_{k} \chi_{I_{j}^{*}}\right)(r) \chi_{I_{j}}(r)\right\|_{l^{q}}^{p_{i}} r^{n-1} d r .
\end{aligned}
$$

Since the intervals $I_{j}$ are mutually disjoint, this is equal to

$$
\begin{aligned}
\frac{c}{\lambda^{p_{i}}} & \sum_{j=-\infty}^{\infty} \int_{I_{j}}\left\|P_{k}^{1}\left(g_{k} \chi_{I_{j}^{*}}\right)(r) \chi_{I_{j}}(r)\right\|_{l^{q}}^{p_{i}} r^{n-1} d r \\
& =\frac{c}{\lambda^{p_{i}}} \sum_{j=-\infty}^{\infty} \int_{I_{j}}\left\|\frac{1}{r^{(n-1) / 2}} \int_{0}^{\infty} K_{k}^{1}(r, s) g_{k}(s) \chi_{I_{j}^{*}}(s) s^{(n-1) / 2} d s \chi_{I_{j}}(r)\right\|_{l^{q}}^{p_{i}} r^{n-1} d r \\
& =\frac{c}{\lambda^{p_{i}}} \sum_{j=-\infty}^{\infty} \int_{I_{j}}\left\|\int_{0}^{\infty} K_{k}^{1}(r, s) g_{k}(s) \chi_{I_{j}^{*}}(s) s^{(n-1) / 2} d s \chi_{I_{j}}(r)\right\|_{l^{q}}^{p_{i}} r^{(n-1)\left(1-p_{i} / 2\right)} d r .
\end{aligned}
$$


Observe now that the weights $R(r)=r^{(n-1)\left(1-p_{i} / 2\right)}$, for $i=0$ and $i=1$, are respectively $r^{(n-1) /(n+1)}$ and $r^{-1}$. So, they are essentially constant on every $I_{j}$, let us call that constant $R\left(2^{j}\right)$, and our estimate is bounded by

$$
\begin{aligned}
\frac{c}{\lambda^{p_{i}}} \sum_{j=-\infty}^{\infty} R\left(2^{j}\right) \int_{I_{j}}\left\|\int_{0}^{\infty} K_{k}^{1}(r, s) g_{k}(s) \chi_{I_{j}^{*}}(s) s^{(n-1) / 2} d s \chi_{I_{j}}(r)\right\|_{l^{q}}^{p_{i}} d r \\
\quad \leq \frac{c}{\lambda^{p_{i}}} \sum_{j=-\infty}^{\infty} R\left(2^{j}\right) \int_{0}^{\infty}\left\|\int_{0}^{\infty} K_{k}^{1}(r, s) g_{k}(s) \chi_{I_{j}^{*}}(s) s^{(n-1) / 2} d s \chi_{I_{j}}(r)\right\|_{l^{q}}^{p_{i}} d r .
\end{aligned}
$$

We know by Theorem 1 in [9] that the operators defined by the kernels $K_{k}^{m}$, $m=1,2,3,4$, are bounded on $L_{l q}^{p}(d r)$ for $4 / 3<p, q<4$. Since $p_{1} \geq 2$ for $n \geq 2$ and also $p_{0} \geq 4 / 3$ for $n>2$, then, for $n>2$, this is less than or equal to

$$
\begin{aligned}
\frac{c}{\lambda^{p_{i}}} \sum_{j=-\infty}^{\infty} R\left(2^{j}\right) \int_{0}^{\infty}\left\|g_{k}(s) \chi_{I_{j}^{*}}(s)\right\|_{l^{q}}^{p_{i}} r^{p_{i}(n-1) / 2} d r \\
\leq \frac{c}{\lambda^{p_{i}}} \sum_{j=-\infty}^{\infty} \int_{I_{j}^{*}}\left\|g_{k}(s) \chi_{I_{j}^{*}}(s)\right\|_{l^{q}}^{p_{i}} r^{p_{i}(n-1) / 2} R(r) d r \\
\leq \frac{3 c}{\lambda^{p_{i}}} \int_{0}^{\infty}\left\|g_{k}(s)\right\|_{l^{q}}^{p_{i}} r^{n-1} d r
\end{aligned}
$$

where we are using that the intervals $I_{j}^{*}$ may overlap at most three of them.

\subsection{Estimate of $\left\{B_{k}\right\}_{k}$ for $n \geq 2$}

Let us observe that if $r \in I_{j}$ and $s \notin I_{j}^{*}$, then $|r-s| \sim r+s$. Then

$$
\begin{aligned}
\left|B_{k}\right| & =\left|\sum_{j=-\infty}^{\infty} \frac{\sigma_{k}(r) \chi_{I_{j}}(r)}{r^{(n-1) / 2}} \int_{\left(I_{j}^{*}\right)^{C}} \frac{g_{k}(s) \mu_{k}(s)}{r-s} s^{(n-1) / 2} d s\right| \\
& \leq c \frac{\left|\sigma_{k}(r)\right|}{r^{(n-1) / 2}} \int_{0}^{\infty} \frac{\left|g_{k}(s) \mu_{k}(s)\right|}{r+s} s^{(n-1) / 2} d s \\
& \leq \frac{c}{r^{(n-1) / 2}} \int_{0}^{\infty} \frac{\left|g_{k}(s) \mu_{k}(s)\right|}{r+s} s^{(n-1) / 2} d s \\
& \leq \frac{c}{r^{(n-1) / 2}}\left\{\int_{\left(I_{k}\right)^{C}} \frac{\left|g_{k}(s) \mu_{k}(s)\right|}{r+s} s^{(n-1) / 2} d s+\int_{I_{k}} \frac{\left|g_{k}(s) \mu_{k}(s)\right|}{r+s} s^{(n-1) / 2} d s\right\} \\
& =B_{k}^{1}+B_{k}^{2} .
\end{aligned}
$$




\subsubsection{Estimate of $\left\{B_{k}^{1}\right\}_{k}$}

First, we observe that $\left|\mu_{k}(s)\right|$ is bounded outside $I_{k}$. Then, by the Minkowski integral inequality we obtain

$$
\left\|B_{k}^{1}\right\|_{l^{q}} \leq \frac{c}{r^{(n-1) / 2}} \int_{0}^{\infty} \frac{\left\|g_{k}(s)\right\|_{l^{q}}}{r+s} s^{(n-1) / 2} d s .
$$

The estimate in $L^{p_{0}, \infty}\left(r^{n-1} d r\right)$ is obtained using that $r^{-(n+1) / 2} \in L^{p_{0}, \infty}\left(r^{n-1} d r\right)=$ $\left(L^{p_{1}, 1}\left(r^{n-1} d r\right)\right)^{*}$. We only need to prove that

$$
\int_{0}^{\infty} G(s) s^{(n-3) / 2} d s \leq c\|G\|_{L^{p_{1}, 1}\left(r^{n-1} d r\right)} .
$$

But this is easy, since we can estimate the left hand side as

$$
\begin{aligned}
\int_{0}^{\infty} G(s) s^{(n-3) / 2} d s & \leq \int_{0}^{\infty} G(s) \frac{1}{s^{(n+1) / 2}} s^{n-1} d s \\
& \leq c\|G\|_{L^{p_{1}, 1}\left(r^{n-1} d r\right)} .
\end{aligned}
$$

Also, we observe that $r^{-(n-1) / 2} \in L^{p_{1}, \infty}\left(r^{n-1} d r\right)=\left(L^{p_{0}, 1}\left(r^{n-1} d r\right)\right)^{*}$, so

$$
\begin{aligned}
\frac{1}{r^{(n-1) / 2}} \int_{0}^{\infty} G(s) s^{(n-3) / 2} d s & \leq \frac{1}{r^{(n+1) / 2}} \int_{0}^{\infty} G(s) \frac{1}{s^{(n-1) / 2}} s^{n-1} d s \\
& \leq \frac{c}{r^{(n+1) / 2}}\|G\|_{L^{p_{0}, 1}\left(r^{n-1} d r\right)} .
\end{aligned}
$$

Thus, we have proved that

$$
\begin{aligned}
\left\|B_{k}^{1}\right\|_{l^{a}} & \leq \frac{c}{r^{(n-1) / 2}}\|\| g_{k}(s)\left\|_{l^{q}}\right\|_{L^{p_{1}, 1}\left(r^{n-1} d r\right)}, \\
\left\|B_{k}^{1}\right\|_{l^{a}} & \leq \frac{c}{r^{(n+1) / 2}}\|\| g_{k}(s)\left\|_{l^{q}}\right\|_{L^{p_{0}, 1}\left(r^{n-1} d r\right)} .
\end{aligned}
$$

Since $r^{-(n-1) / 2} \in L^{p_{1}, \infty}\left(r^{n-1} d r\right)$ and $r^{-(n+1) / 2} \in L^{p_{0}, \infty}\left(r^{n-1} d r\right)$, we obtain

$$
\begin{aligned}
& \left\|B_{k}^{1}\right\|_{L_{l q}^{p_{1}, \infty}\left(r^{n-1} d r\right)} \leq c\left\|g_{k}(s)\right\|_{L_{l q}^{p_{1}, 1}\left(r^{n-1} d r\right)}, \\
& \left\|B_{k}^{1}\right\|_{L_{l q}^{p_{0}, \infty}\left(r^{n-1} d r\right)} \leq c\left\|g_{k}(s)\right\|_{L_{l q}^{p_{0}, 1}\left(r^{n-1} d r\right)} .
\end{aligned}
$$

\subsubsection{Estimate of $\left\{B_{k}^{2}\right\}_{k}$}

By the Hölder inequality we obtain, for some $\alpha>1$,

$$
B_{k}^{2} \leq \frac{c}{r^{(n-1) / 2}}\left(\int_{I_{k}} \frac{\left|g_{k}(s)\right|^{\alpha}}{|r+s|^{\alpha}} s^{\alpha(n-1) / 2} d s\right)^{1 / \alpha}\left(\int_{I_{k}}\left|\mu_{k}(s)\right|^{\alpha^{\prime}} d s\right)^{1 / \alpha^{\prime}},
$$


where $\alpha^{\prime}=\frac{\alpha}{\alpha-1}$ is the conjugate exponent of $\alpha$. Now, following the estimates (1) and (2), for $s>0$

$$
\mu_{k}(s) \leq c\left(k^{-1 / 6}+\left|\frac{s-k}{s+k}\right|^{1 / 4}\right)^{-1}=w
$$

and notice that the weight $w^{\alpha^{\prime}}$ is in the Muckenhoupt class $A_{1}$ for $\alpha^{\prime}<4$, which means $\alpha>\frac{4}{3}$ (see [5]). So, $M\left(w^{\alpha^{\prime}}\right) \leq c w^{\alpha^{\prime}}$, where $M$ is the Hardy-Littlewood maximal operator. Then, for $\alpha>\frac{4}{3}$ we have

$$
\left(\int_{I_{k}}\left|\mu_{k}(s)\right|^{\alpha^{\prime}} d s\right)^{1 / \alpha^{\prime}} \leq c\left(\int_{I_{k}}\left(k^{-1 / 6}+\left|\frac{s-k}{s+k}\right|^{1 / 4}\right)^{-\alpha^{\prime}} d s\right)^{1 / \alpha^{\prime}} \leq c k^{1 / \alpha^{\prime}} .
$$

Let us observe now that we can introduce in the integral the constant $k$ as the variable $s$ because we are integrating in the interval $I_{k}$, obtaining

$$
\begin{aligned}
B_{k}^{2} & \leq \frac{c}{r^{(n-1) / 2}}\left(\int_{I_{k}} \frac{\left|g_{k}(s)\right|^{\alpha}}{|r+s|^{\alpha}} s^{\alpha(n-1) / 2} s^{\alpha / \alpha^{\prime}} d s\right)^{1 / \alpha} \\
& =\frac{c}{r^{(n-1) / 2}}\left(\int_{I_{k}} \frac{\left|g_{k}(s)\right|^{\alpha}}{|r+s|^{\alpha}} s^{\alpha(n+1) / 2} \frac{d s}{s}\right)^{1 / \alpha} .
\end{aligned}
$$

The $l^{q}$-norm is then

$$
\left\|B_{k}^{2}\right\|_{l^{q}} \leq \frac{c}{r^{(n-1) / 2}}\left\|\int_{I_{k}} \frac{\left|g_{k}(s)\right|^{\alpha}}{|r+s|^{\alpha}} s^{\alpha(n+1) / 2} \frac{d s}{s}\right\|_{l^{q / \alpha}}^{1 / \alpha} .
$$

This expression only makes sense for $q / \alpha \geq 1$, that is, for $\alpha \leq q$. If now we use the Minkowski inequality we obtain

$$
\left\|B_{k}^{2}\right\|_{l^{q}} \leq \frac{c}{r^{(n-1) / 2}}\left(\int_{I_{k}} \frac{\left\|g_{k}(s)\right\|_{l^{q}}^{\alpha}}{|r+s|^{\alpha}} s^{\alpha(n+1) / 2} \frac{d s}{s}\right)^{1 / \alpha} .
$$

Let us call now $G=\left\|g_{k}(s)\right\|_{l^{q}}$ and define the operators $D_{\alpha}$ by

$$
D_{\alpha}(G)=\frac{1}{r^{(n-1) / 2}}\left(\int_{I_{k}} \frac{|G|^{\alpha}}{|r+s|^{\alpha}} s^{\alpha(n+1) / 2} \frac{d s}{s}\right)^{1 / \alpha} .
$$

We want to prove that $D_{\alpha}$ is bounded when acting between the spaces

$$
D_{\alpha}: L^{p_{i}, 1}\left(r^{n-1} d r\right) \longrightarrow L^{p_{i}, \infty}\left(r^{n-1} d r\right),
$$

for $i=0,1$ and some exponent $\alpha \in(4 / 3, q]$. 
For the estimate corresponding to $p_{0}$ we choose $\alpha=p_{0}$ and extract the $|r+s|$ out of the integral as a constant times $r$ :

$$
\begin{aligned}
D_{p_{0}}(G) & =\frac{1}{r^{(n-1) / 2}}\left(\int_{I_{k}} \frac{|G|^{p_{0}}}{|r+s|^{p_{0}}} s^{p_{0}(n+1) / 2} \frac{d s}{s}\right)^{1 / p_{0}} \\
& \leq \frac{c}{r^{(n+1) / 2}}\left(\int_{I_{k}}|G|^{p_{0}} s^{n-1} d s\right)^{1 / p_{0}} \\
& \leq \frac{c}{r^{(n+1) / 2}}\|G\|_{L^{p_{0}, 1}\left(r^{n-1} d r\right)} .
\end{aligned}
$$

Since $r^{-(n+1) / 2} \in L^{p_{0}, \infty}\left(r^{n-1} d r\right)$, we have proved that $D_{p_{0}}$ is bounded when acting between the spaces

$$
D_{p_{0}}: L^{p_{0}, 1}\left(r^{n-1} d r\right) \longrightarrow L^{p_{0}, \infty}\left(r^{n-1} d r\right) .
$$

We consider now the exponent $p_{1}$. First of all, we change in the integral $|r+s|$ by $s$, so

$$
D_{\alpha}(G) \leq \frac{c}{r^{(n-1) / 2}}\left(\int_{I_{k}}|G|^{\alpha} s^{\alpha(n-1) / 2} \frac{d s}{s}\right)^{1 / \alpha} .
$$

Then recall that $r^{-(n-1) / 2} \in L^{p_{1}, \infty}\left(r^{n-1} d r\right)$, so we only have to prove that

$$
\left(\int_{I_{k}}|G|^{\alpha} s^{\alpha(n-1) / 2} \frac{d s}{s}\right)^{1 / \alpha} \leq c\|G\|_{L^{p_{1}, 1}\left(r^{n-1} d r\right)} .
$$

We can't choose $\alpha=p_{1}$, because for $n=2$ it is outside the interval $(4 / 3, q]$. But for $2 \leq q \leq p_{1}$ we can consider $\alpha=2$, and to prove the corresponding inequality we only observe that $r^{-1} \in L^{n, \infty}\left(r^{n-1} d r\right)$ and that $\left(L^{n /(n-1), 1}\left(r^{n-1} d r\right)\right)^{*}=L^{n, \infty}\left(r^{n-1} d r\right)$ because $n$ and $\frac{n}{n-1}$ are conjugate exponents, so

$$
\left(\int_{I_{k}}|G|^{2} s^{n-1} \frac{d s}{s}\right)^{1 / 2} \leq c\left\|G^{2}\right\|_{L^{n /(n-1), 1}\left(r^{n-1} d r\right)}^{1 / 2}=c\|G\|_{L^{p_{1}, 1}\left(r^{n-1} d r\right)} .
$$

For the case $p_{0}<q<2$ we choose $\alpha=q$ and, since $I_{k}=\left[2^{k}, 2^{k+1}\right)$ with $k \geq 1$, we can estimate

$$
\left(\int_{I_{k}}|G|^{q} s^{q(n-1) / 2} \frac{d s}{s}\right)^{1 / q} \leq\left(\int_{I_{k}}|G|^{q} s^{n-1} \frac{d s}{s}\right)^{1 / q} .
$$

Now, observe that $r^{-1} \in\left(L^{p_{1} / q, 1}\left(r^{n-1} d r\right)\right)^{*}=L^{\left(p_{1} / q\right)^{\prime}, \infty}\left(r^{n-1} d r\right)$, and obtain

$$
\begin{aligned}
\left(\int_{I_{k}}|G|^{q} s^{n-1} \frac{d s}{s}\right)^{1 / q} & \leq\left(\left\|G^{q}\right\|_{L^{p_{1} / q, 1}\left(r^{n-1} d r\right)}\left\|r^{-1}\right\|_{L^{\left(p_{1} / q\right)^{\prime}, \infty}\left(r^{n-1} d r\right)}\right)^{1 / q} \\
& \leq c\left\|G^{q}\right\|_{L^{p_{1} / q, 1}\left(r^{n-1} d r\right)}^{1 / q}=c\|G\|_{L^{p_{1}, 1}\left(r^{n-1} d r\right)} .
\end{aligned}
$$

This ends our proof. 
It is remarkable that the case $n=2$ in this proof is the hardest, as happens in [1]. This seems to be in the philosophy of the article [10].

\section{References}

[1] A. Carbery, E. Romera, and F. Soria, Radial weights and mixed norm inequalities for the disc multiplier, J. Funct. Anal. 109 (1992), no. 1, 52-75.

[2] L. Carleson, On convergence and growth of partial sums of Fourier series, Acta Math. 116 (1966), 135-157.

[3] A. Córdoba, The disc multiplier, Duke Math. J. 58 (1989), no. 1, 21-29.

[4] C. Fefferman and E. M. Stein, Some maximal inequalities, Amer. J. Math. 93 (1971), 107-115.

[5] J. García-Cuerva and J. L. Rubio de Francia, Weighted norm inequalities and related topics, North-Holland Mathematics Studies, vol. 116, North-Holland Publishing Co., Amsterdam, 1985.

[6] R. A. Hunt, On the convergence of Fourier series, Orthogonal Expansions and their Continuous Analogues (Edwardsville, Ill., 1967), Southern Illinois Univ. Press, Carbondale, Ill., 1968, pp. 235-255.

[7] G. Mockenhaupt, On radial weights for the spherical summation operator, J. Funct. Anal. 91 (1990), no. 1, 174-181.

[8] E. Romera, Convergencia esférica de integrales de Fourier: Nuevas estimaciones para el operador multiplicador del disco, tesis doctoral, Universidad Autónoma de Madrid, 1991.

[9] _ Vector valued inequalities for Hankel transforms, J. London Math. Soc. (2) 51 (1995), no. 2, 299-308.

[10] J. L. Rubio de Francia, Transference principles for radial multipliers, Duke Math. J. 58 (1989), no. $1,1-19$.

[11] E. M. Stein, Some problems in harmonic analysis, Harmonic analysis in Euclidean spaces (Williams Coll., Williamstown, Mass., 1978), Proc. Sympos. Pure Math., vol. 35, part I, Amer. Math. Soc., Providence, R.I., 1979, pp. 3-20.

[12] E. M. Stein and G. Weiss, Introduction to Fourier analysis on Euclidean spaces, Princeton Mathematical Series, vol. 32, Princeton University Press, Princeton, N.J., 1971.

[13] G. N. Watson, A treatise on the theory of Bessel functions, Cambridge Mathematical Library, Cambridge University Press, Cambridge, 1995. Reprint of the second (1944) edition. 